EESTI NSV TEADUSTE AKADEEMIA TOIMETISED 1955, IV kd., nr. 4 ИЗВЕСТИЯ АКАДЕМИИ НАУК ЭСТОНСКОИ ССР 1955. Том IV, № 4

\title{
К ТЕПЛОВОМУ РАСЧЕТУ ПРОЦЕССА ПОЛУКОКСОВАНИЯ СЛАНЦА В ШАХТНОМ ГЕНЕРАТОРЕ
}

\author{
М. Я. ГУБЕРГРИЦ, \\ кандидат технических наук
}

Метод термической переработки сланца в шахтных генераторах основан на использовании тепла горения твердого остатка для газопротивоточного полукоксования сланца. В организации технологического процесса ведущую роль играют, таким образом, условия передачи тепла от парогазового теплоносителя к сланцу в шахте полукоксования. Этопредположение подтверждается результатами изучения технологического режима и условий газообразования в генераторе современного типа $\left({ }^{4,5}\right)$.

Для определения путей дальнейшего совершенствования метода чрезвычайно важно найти достоверный способ оценки интенсивности процесса теплоотдачи в шахте перегонки. Рассмотрению этой задачи в свете современных научных воззрений и посвящено настоящее сообщение.

\section{Способы решения внешней задачи при оценке интенсивности теплообмена в шахте полукоксования}

Передача тепла от парогазового теплоносителя к сланцу в.шахте полукоксования осуществляется путем конвекции, теплопроводности и лучеиспускания. В силу значительной скорости движения газового потока, основное количество тепла передается сланцу благодаря вынужденной конвекции. Относительная роль остальных видов теплообмена невелика и пренебрежение их влиянием не вызывает существенной погрешности в результатах практических расчетов $(10,15)$.

Следовательно, основным критерием оценки интенсивности «газопротивоточного» теплообмена ${ }^{1}$ будет служить величина коэффициента теплоотдачи $\alpha$ ккал $/ \mathrm{M}^{2}$. час. град. При определении этой величины для конвективного теплообмена в кусковом слое следует учитывать влияние ряда факторов. Наиболее существенными из них являются: гидродинамическая характеристика системы, температурные условия, теплофизические свойства и состав парогазового теплоносителя, структурная и теплофизическая характеристика слоя (гранулометрический состав, суммарная порозность, размеры, форма и шероховатость кусков, суммарная и активная поверхность слоя и т. д.).

1 Применение этого термина, несмотря на его неточность, представляется наиболее удобным. 
Решение этой задачи чисто математическим путем весьма сложно; влияние многих факторов может быть установлено лишь в результате опыта. В зависимости от того, какого рода элементы процесса подлежат экспериментальному изучению, существующие методы определения величины коэффициента теплоотдачи следует, с известной условностью, подразделить на две основные группы.

В методах, основанных на решении «внешней» задачи, в явном виде учитываются условия движения и свойства теплоносителя. Характеристика взаимодействующего с ним кускового слоя учитывается главным образом в неявной форме - в виде эмпирических коэффициентов.

Величина внутреннего термического сопротивления куска, обусловленная его размерами и теплофизическими свойствами материала, при этом не может быть учтена. Таким образом, приводимые ниже уравнения внешней задачи могут быть использованы лишь в частных случаях для конвективной теплоотдачи к мелкозернистому материалу либо кускам, обладающим высокой теплопроводностью.

В самом общем виде процесс вынужденного конвективного теплообмена в слое (при использовании этого принципа) описывается следующим уравнением, выраженным в критериальной форме:

$$
N u=A \mathrm{Re}^{h} .
$$

Здесь $N u=a d_{c p} / \lambda$ и $\mathrm{Re}=W d_{c p} / v$, где $d_{c p}$ - средний диаметр куска, $W$, 2. и $\boldsymbol{\nu}$-соответственно, линейная скорость и коэффициенты теплопроводности и кинематической вязкости теплоносителя. Таким образом, в общем виде

$$
a=A \frac{\lambda}{d_{\text {cp }}} \mathrm{Re}^{h} .
$$

Величины эмпирических коэффициентов $A$ и $h$ отыскиваются опытным путем для соответствующих определяющих условий. В данном случае такими условиями являются порозность слоя и форма частиц.

В связи с этим не представляется необходимым подробно останавливаться на результатах ряда исследований - С. Фурнаса $\left({ }^{18}\right)$, Р. С. Бернштейна $\left({ }^{2}\right), 3$. Ф. Чуханова и Е. А. Шапатиной $\left({ }^{16}\right)$, И. М. Федорова $\left({ }^{14}\right)$, А. Ф. Чудновского $\left({ }^{15}\right)$ и других. Следует указать, что по некоторым данным $\left({ }^{2}\right)$ для кусков неправильной формы показатель степени $h=1$.

В. Н. Тимофеев $\left({ }^{12}\right)$ в результате обобщения и обработки значительного количества экспериментальных данных (для кусков любой формы и различной крупности) рекомендует применять следующие уравнения с учетом гидродинамического состояния системы.

В пределах значений $\mathrm{Re}$ от 20 до 200

$$
N u=0,106 \operatorname{Re} \text { и } a=0,106 W \frac{\lambda}{\nu} .
$$

Для $\mathrm{Re}>200$ :

$$
N u=0,61 \mathrm{Re}^{0,67} \text { и } \alpha=0,61 \lambda \frac{W^{0,67}}{d_{\mathrm{c} p}^{0,33} v^{0,67}} .
$$

В. Н. Тимофеев отрицает влияние порозности слоя на величину коэффициента теплоотдачи.

По существу, уравнениями (3) и (3a) можно ограничить перечень способов решения внешней задачи.

Результаты расчетного определения величины $\alpha$ указанными методами могут быть использованы в настоящем случае для проверки данных, полученных при решении внутренней задачи. Числовое значение последних должно быть обязательно ниже величины $\alpha$, определенной без учета термического сопротивления кусков. 


\section{Способы решения внутренней задачи}

Метод решения внутренней задачи применительно к шахтным печам разработан Б. И. Китаевым $\left({ }^{7,8}\right)$, который использовал результаты гидравлического моделирования тепловых явлений для обработки данных С. Фурнаса и других исследователей. В расчетных уравнениях учитываются форма, размеры и термическое сопротивление кусков материала, а также порозность слоя.

Для слоя кусков, обладающих бесконечно большой теплопроводностью, объемный коэффициент теплоотдачи

$$
a_{\mathbf{v}}=A \frac{W_{0}^{0,9} T^{0,3}}{d^{0,75}} m_{1} \quad \text { ккал/M }{ }^{3} \text {. час. град. }
$$

Здесь $A$ - эмпирический коэффициент - составляет для ряда материалов (кокс, уголь, известняк и др.) примерно одну и ту же величину в пределах 166-170; скорость теплоносителя $W_{0}$ отнесена к нормальным условиям и полному сечению шахты и выражается в м/сек.; $m_{1}-$ коэффициент, учитывающий влияние порозности слоя на процесс теплопередачи.

Определение $a_{v}$ с учетом термического сопротивления куска заданной формы, радиуса $r$ и теплопроводности $\lambda_{c}$ производится по уравнению:

$$
a_{\mathrm{v} \varepsilon}=\frac{\alpha_{\mathrm{v}}}{1+\frac{1}{K_{\phi}} \frac{\alpha \mathrm{r}}{\lambda_{\mathrm{c}}}}
$$

где $K_{\phi}-$ «тепловой» коэффициент формы куска (изменяется от 3 до 3,5 и 5 , соответственно, для плиты, цилиндра и шара); $\alpha-$ коэффициент теплоотдачи, отнесенный к единице поверхности слоя $\alpha=\frac{\boldsymbol{c}_{\mathrm{v}}}{S_{\mathrm{yд}}}$, где $S_{\mathrm{yд}}$ удельная поверхность слоя в $\mathrm{M}^{2} / \mathrm{M}^{3}$. Истинная величина $\alpha=\alpha_{\varepsilon}$ определится из уравнения

$$
a=\frac{\alpha_{\mathrm{v} \varepsilon}}{S_{\mathrm{yA}}}
$$

Вторым методом ориентировочного решения внутренней задачи может явиться анализ теплового баланса системы. Количество тепла, воспринимаемого сланцем в единицу времени, составляет:

$$
Q_{\tau}=a S_{\varepsilon} \Delta t_{\text {тс }} \quad \text { ккал/час; }
$$

где $Q_{\tau}$ - часовой приход тепла; $S_{\varepsilon}$ - величина суммарной поверхности слоя $\left(\mathrm{M}^{2}\right)$ и $\Delta t_{\mathrm{тc}}$ - температурный напор в системе, т. е. средняя по высоте слоя разность температур теплоносителя и сланца. Проще оперировать не с суммарной поверхностью, а с величиной удельной поверхности слоя

$$
S_{\mathrm{y} \lambda}=S=\frac{S_{\varepsilon}}{V_{\mathrm{c}}}=\frac{S_{\varepsilon}}{G_{\mathrm{c}}} \gamma_{\mathrm{H}},
$$

где $\gamma_{\text {н }}-$ насыпной вес топлива; $G_{\mathbf{c}}-$ весовой расход его в единицу времени. 
В результате подстановки уравнение (7) приобретает вид:

$$
Q \tau=a S \frac{G_{\mathrm{c}}}{\gamma_{\mathrm{H}}} \Delta t_{\mathrm{\tau c}} .
$$

С другой стороны, $Q_{\tau}=Q_{\mathrm{c}} \cdot G_{\mathrm{c}}$, где $Q_{\mathrm{c}}$ - расход тепла на переработку весовой единицы сланца. Эта величина определится из общего уравнения теплового баланса теплоносителя:

$$
Q_{\mathrm{T}}=Q_{\mathrm{c}}+Q_{\mathrm{n}}+Q_{\text {ок }} \quad \text { ккал } / \kappa г,
$$

где $Q_{\mathrm{T}}$ - теплосодержание парогазового теплоносителя на входе в шахту полукоксования; $Q_{\mathrm{n}}$ - потери тепла с уходящим теплоносителем; $Q_{\text {ок }}$ потери тепла в окружающую среду. Из уравнения (9) определяется величина $Q_{\mathrm{c}}$ :

$$
Q_{\mathrm{c}}=\left(Q_{\mathrm{T}}-Q_{\mathrm{n}}\right)-Q_{\mathrm{o \kappa}} \text {. }
$$

При этом $\left(Q_{\tau}-Q_{n}\right)=G_{\tau} c_{\mathrm{p}}^{\mathrm{T}} \Delta t_{\tau}$, где $G_{\tau}-$ удельный расход теплоносителя (на весовую единицу перерабатываемого сланца), $c_{\mathrm{p}}^{\mathrm{T}}-$ его теплоемкость при данной температуре, $\Delta t_{\mathrm{T}}$ - перепад температуры теплоносителя в слое.

Таким образом,

$$
Q_{\mathrm{c}}=\left(G_{\mathrm{\tau}} c_{\mathrm{p}}^{\mathrm{T}} \Delta t_{\mathrm{\tau}}-Q_{\mathrm{o \kappa}}\right) \quad \text { и } \quad Q_{\tau}=\left(G_{\mathrm{\tau}} c_{\mathrm{p}}^{\mathrm{T}} \Delta t_{\mathrm{T}}-Q_{\mathrm{o \kappa}}\right) \cdot G_{\mathrm{c}} .
$$

Из (8) и (11), после несложных преобразований, получаем:

$$
a \frac{S}{\gamma_{\mathrm{H}}} \Delta t_{\mathrm{\tau c}}=\left(G_{\mathrm{\tau}} c_{\mathrm{p}}^{\mathrm{\tau}} \Delta t_{\mathrm{\tau}}-Q_{\mathrm{o \kappa}}\right) \quad \text { или } \quad a=\frac{\left(G_{\mathrm{\tau}} c_{\mathrm{p}}^{\top} \Delta t_{\mathrm{\tau}}-Q_{\mathrm{o \kappa}}\right)}{S \cdot \Delta t_{\mathrm{Tc}}} \gamma_{\mathrm{H}} .
$$

На использовании аналогичного уравнения были основаны опыты ряда исследователей, упомянутых выше, а также первая попытка анализа тепловых явлений, протекающих в генераторе (').

Практическое решение уравнений (4)-(6) и (12) связано с известными трудностями, так как в этих целях необходимо располагать хотя бы ориентировочными, но достоверными данными о величине активной удельной поверхности слоя. Так, С. Фурнас полагает нецелесообразным рассчитывать величину $\alpha$ на единицу поверхности слоя ввиду очевидных трудностей в определении последней и особенно в определении доли суммарной поверхности, активно участвующей в процессе теплопередачи.

\section{Определение удельной поверхности насыпного материала}

Общепринятый метод определения величины удельной «макроповерхности» слоя шарообразных частиц основывается на следующем положении:

$$
S=n S_{\kappa}=\frac{(1-m) S_{\kappa}}{V_{\kappa}},
$$

где $S_{\kappa}$ и $V_{\kappa}-$ соответственно, поверхность и объем одного куска, $n=\frac{(1-m)}{V_{k}}-$ число кусков в единице объема слоя. 
Суммарная величина удельной поверхности для монофракционного слоя

$$
S=\frac{6(1-m)}{d}
$$

н для полифракционного слоя

$$
S=\frac{6(1-m)}{d_{\mathrm{cp}}}
$$

При рассмотрении слоя, состоящего из реальных частиц, представляется целесообразным установить некоторый поправочный коэффициент, так называемый коэффициент формы, с введением которого учитывается отличие формы данного куска от шарообразной. Автором настоящей статьи показано $\left({ }^{3}\right)$, что куски технологического сланца в диапазоне крупности от 10 до 100 мм по своей форме в пределе приближаются к сплющенному цилиндру с эллиптическим сечением. Основные размеры таких частиц могут быть выражены через средний диаметр следующим образом: $H=1,45 d_{\mathrm{cp}} ; \quad R=0,965 d_{\mathrm{cp}} ; \quad r=0,582 d_{\mathrm{cp}}$. Поверхность частицы $S_{\mathrm{u}}=$ $=1,40 \pi d_{\mathrm{cp}}^{2}$ и объем $V_{\text {и }}=0,64 d_{\mathrm{cp}}^{3}$. Отнеся эти величины к соответствующим показателям для шара $\left(S_{\mathrm{m}}=\pi d_{\mathrm{cp}}^{2}, \quad V_{\mathrm{m}}=0,524 d_{\mathrm{cp}}^{3}\right)$, получаем «частные» коэффициенты формы для кускового технологического сланца

$$
K_{\mathrm{S}}=S_{\mathrm{II}}: S_{\mathrm{II}}=1,40 ; \quad K_{\mathrm{V}}=V_{\mathrm{u}}: V_{\mathrm{ul}}=1,20
$$

Вводим в уравнение (13) суммарный коэффициент формы $K_{\phi}=K_{\mathrm{s}}: K_{\mathrm{v}} \approx$ $\approx 1,165$. Тогда уравнение (13) принимает вид:

$$
S=1,165 \frac{6(1-m)}{d_{\mathrm{cp}}}=\frac{7(1-m)}{d_{\mathrm{cp}}}
$$

Х. К. Труу $\left({ }^{13}\right)$, изучая структурные свойства кускового сланца, установил величины поверхности и объема кусков $S_{\kappa}=3,76 b^{2}$ и $V_{\kappa}=0,38 b^{3}$, где $b$ - поперечник куска $\left(b=R=0,965 d_{\mathrm{cp}}\right)$. На основании (13) величина удельной поверхности слоя

$$
S=\frac{(1-m) 3,76 b^{2}}{0,38 b^{3}}=\frac{9,9(1-m)}{d_{\mathrm{cp}}}
$$

Б. И. Китаев $\left({ }^{7,8}\right)$ в расчетах, связанных с определением коэффициента теплоотдачи в доменной печи, применяет формулу

$$
S=\frac{7,5(1-m)}{d_{\mathrm{cp}}}
$$

Для полидисперсного материала (доменной шихты) с пределами крупности 25-100 мм К. И. Сысков (11) рекомендует употреблять формулу

$$
S=0,06 K_{\phi} \sum \frac{a}{\gamma_{\mathrm{n}} d_{\mathrm{cp}}}
$$

где $K_{\phi}$ - коэффициент формы, $a$ - весовой выход фракции в процентах, $\gamma_{\text {н }}$ и $d_{\text {ср }}-$ соответствующие показатели для каждой фракции. 
Все приведенные выше уравнения типа (13) позволяют определить расчетным путем величину удельной поверхности кускового слоя с известной, иногда весьма значительной, погрешностью. Вследствие контактирования кусков неправильной формы, а зачастую из-за склонности некоторых материалов к слеживанию, величина активной поверхности теплообмена может существенно отличаться от определенной расчетным путем.

Для сильно слеживающихся материалов (кусковой торф, древесная щепа) Д. Б. Гинзбург $\left({ }^{6}\right)$ использует известную формулу

$$
S=\frac{3,5(1-m)}{d_{\mathrm{cp}}^{0,65}} .
$$

Для древесного угля применима формула

$$
S=\frac{3,77}{d_{\mathrm{cp}}}=\frac{6,85(1-m)}{d_{\mathrm{cp}}} \text { при } m \approx 0,45 .
$$

Таким образом, для каждого материала и конкретных условий его переработки в слое следует уточнить расчетные формулы типа (13). Ниже приводятся результаты определения величины $S$ для технологического сланца по различным вариантам; они подлежат далее количественной проверке путем сравнения значений $\alpha$ из уравнений $(2)-(3),(4)-(6)$ и (12).

\section{Исходные данные для расчетного определения коэффициента теплоотдачи в шахте полукоксования}

Основой для проведения проверочных расчетов являются результаты балансового испытания группы генераторов $\left({ }^{4,5}\right)$. В настоящем разделе приводятся исходные данные для расчета; промежуточные расчетные операции опускаются во избежание загромождения статьи излишними подробностями.

Температурный режим в шахте полукоксования характеризуется следующими ориентировочными показателями:

$\begin{array}{rrrr}\text { Tемпература теплоносителя на входе в шахту . } & 620^{\circ} \\ , & \text { на выходе из шахты } & . & 195^{\circ} \\ , & \text { сланца в верхней части слоя . . . . . } & 20^{\circ} \\ , & \text { на выходе из шахты . . . . . . . } & 450^{\circ}\end{array}$

Соответственно, перепад температуры теплоносителя в слое $\Delta t_{\mathrm{T}}=$ $=425^{\circ}$; средняя величина температурного напора $\Delta t_{\tau c}=175^{\circ}$; средняя рабочая температура теплоносителя $\left({ }^{10}\right) t_{\mathrm{cp}}=400^{\circ}$.

В таблице 1 приведены исходные данные для приближенного расчета скорости теплоносителя; значительную долю в общем расходе его составляет водяной пар. Это учитывается при определении величин $\lambda$ и $v$ при

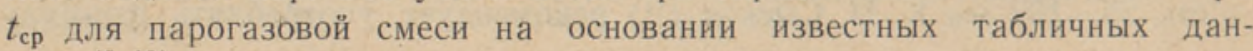
ных $(9,10)$.

Средняя линейная скорость теплоносителя при нормальных условиях (на полное сечение шахты в средней ее части) $W_{0}$ составляет $505 \mathrm{~m} /$ час $=0,141 \mathrm{~m} / \mathrm{cek}$. Предполагается, что газообразная часть теплоносителя по составу идентична суммарному газу генераторов; объем паров смолы и их свойства не учитываются. 
Состав и удельный расход теплоносителя

\begin{tabular}{|c|c|c|c|}
\hline $\begin{array}{c}\text { Наименование } \\
\text { компонентов }\end{array}$ & $\begin{array}{c}\text { Удельный } \\
\text { расход } \\
\text { в нм }{ }^{3} / \mathrm{kr}\end{array}$ & $\begin{array}{c}\text { Наименование } \\
\text { компонентов }\end{array}$ & $\begin{array}{c}\text { удельный }_{\text {дасход }} \\
\text { в нм } 3 / \text { кr }\end{array}$ \\
\hline $\begin{array}{l}\text { Газы } \\
\text { Продукты горения, сухие } \\
\mathrm{CO}_{2} \text { разложения карбо- } \\
\text { натов } \\
\text { Обратный газ } \\
\text { Газ полукоксования (ча- } \\
\text { стично) }\end{array}$ & $\begin{array}{l}0,424 \\
0,103 \\
0,140 \\
0,020\end{array}$ & $\begin{array}{l}\text { Пары воды } \\
\text { Пар дутьевой и влага } \\
\text { дутья } \\
\text { Влага горения }\end{array}$ & $\begin{array}{l}0,286 \\
0,080\end{array}$ \\
\hline Итого газов & 0,687 & Итого паров воды & 0,366 \\
\hline
\end{tabular}

Определение удельной поверхности слоя пронзводится на основании данных таблицы 2.

Таблица 2

Гранулометрический состав и размерная характеристика сланца

\begin{tabular}{|c|c|c|c|c|c|c|}
\hline \multirow{2}{*}{$\begin{array}{c}\text { Наименование } \\
\text { показателей }\end{array}$} & \multicolumn{6}{|c|}{ Класс крупности в мм } \\
\hline & $0-15$ & $15-25$ & $25-38$ & $38-60$ & $60-100$ & $\mid 100-150$ \\
\hline $\begin{array}{l}\text { Выход фракций, вес. \% } \\
\text { Средний диаметр куска, } \\
\text { мм }\end{array}$ & $\begin{array}{l}5,2 \\
7\end{array}$ & $\begin{array}{r}6,4 \\
21\end{array}$ & $\begin{array}{l}36,1 \\
34\end{array}$ & $\begin{array}{l}24,7 \\
46\end{array}$ & $\begin{array}{l}24,8 \\
72\end{array}$ & $\begin{array}{r}2,6 \\
112\end{array}$ \\
\hline
\end{tabular}

Средний диаметр куска для технологического сланца $d_{\mathrm{cp}}=46 \mathrm{мм}=$ $=0,046 \mathrm{м} ;$ насыпной вес $\gamma_{\text {н }}=0,9 \mathrm{~T} / \mathrm{M}^{3} ;$ порозность слоя $m \approx 0,4$.

Результаты вычисления величины $S$ по различным формулам сведены в таблице 3.

Результаты расчетного определения удельной поверхности сланца

$S=\frac{6(1-m)}{d_{\text {ср }}}$ для слоя шарообразных частиц

$S=1,165 \frac{6(1-m)}{d_{\text {ср }}}$ для слоя реальных частиц

$S=0,06 \sum \frac{a}{\gamma_{\mathrm{H}} d_{\mathrm{cp}}}$ по К. И. Сыскову

То же, с учетом коэффициента формы $K_{\phi}=1,165$

$S=\frac{3,5(1-m)}{d_{\text {cp }}^{0,65}}$ для слеживающйся материалов

То же, с учетом коэффициента формы

$S=\frac{9,9(1-m)}{d_{\mathrm{cp}}}$ по Х. К. Труу 


\section{Расчетное определение коэффициента теплоотдачи}

Для определения $a$ на основании (3) и (3a) подсчитывается величина числа Re; в данных условиях она примерно равна 200. Поэтому для переходной области целесообразно произвести расчет по обоим вариантам.

По уравнению (3) при $\mathrm{Re} \leqslant 200$;

$$
\alpha=0,106 \cdot 215=23 \text { ккал } / \mathrm{M}^{2} \text {. час. град. }
$$

Для $\operatorname{Re}>200$ :

$$
a=0,61 \cdot 0,05 \cdot \frac{117,5}{0,426 \cdot 0,362}=23,2 \text { ккал } / \mathrm{M}^{2} . \text { час. град. }
$$

При расчете по системе уравнений (4)-(6) основным затруднением является выбор величины активной удельной поверхности материала. Це-

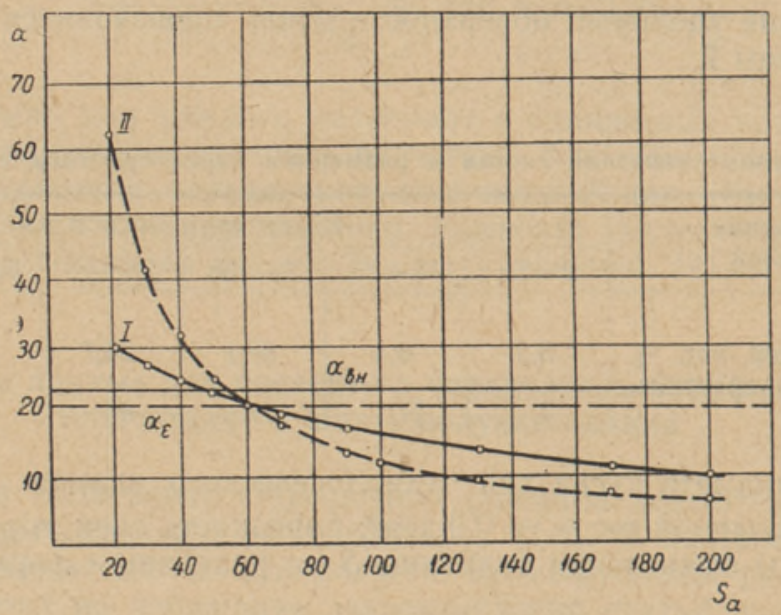

Рис. 1. Зависимость коэффициента теплоотдачи от удельной поверхности слоя. I - кривая по уравнениям (4) - (6); II - кривая уравнения теплового баланса (12); $\alpha_{\text {өн }}$ - значение коэффициента теплоотдачи, вычисленное по методу решения внешней задачи; $\alpha_{\varepsilon}-$ значение коэффициента теплоотдачи для реальных условий.

лесообразно произвести расчет для диапазона значений величин $S$, приведенных в таблице $3\left(20-200 \mathrm{~m}^{2} / \mathrm{m}^{3}\right)$, и сравнить полученные данные с результатами определения $a$ по уравнению (3) и уравнениям теплового баланса.

Согласно (4)

$$
a_{v}=166 \cdot \frac{0,141^{0,9} \cdot 673^{0,3}}{0,046^{0,75}} 1,4=2840 \text { ккал/ } / \mathrm{M}^{3} . \text { час. град. }
$$

При определении $\alpha_{v \varepsilon}$ следует принять: $K_{\Phi}=3,5$ (для тел цилиндрической формы) и $\lambda_{\mathrm{c}}=0,25$ ккал/м. час. град. - на основании данных К. Лутса $\left({ }^{19}\right)$ и более поздних сообщений $\left({ }^{17}\right)$.

Результаты подстановки различных значений $S$ в уравнения (5) и (6), т. е. зависимость величины $\alpha$ от активной поверхности теплоотдачи, при прочих равных условиях, интерпретированы графически в виде кривой I на рис. 1. 
При подстановке соответствующих исходных данных в уравнение теплового баланса (12) получаем:

$$
a=\frac{\left(G_{\mathrm{T}} c_{\mathrm{p}}^{\mathrm{T}} \Delta_{\mathrm{T}}^{\prime}-Q_{\mathrm{o \kappa}}\right) \gamma_{\mathrm{H}}}{S \cdot \Delta t_{\mathrm{rc}}}=\frac{(274-30) \cdot 900}{S \cdot 175}=\frac{1255}{S_{\mathrm{a}}} \text { ккал } / \mathrm{M}^{2} . \text { час. град. }
$$

Зависимость значений $\alpha$ от величины $S$ в указанном диапазоне приведена на рис. 1 в виде кривой II.

Точка пересечения кривых I и II должна соответствовать наиболее вероятным значениям величин $\alpha$ и $S_{\text {a }}$ при данных конкретных условиях. Величина активной удельной поверхности слоя при этом составляет $S_{\mathrm{a}}=$ $=60 \mathrm{~m}^{2} / \mathrm{M}^{3}$, а коэффициент теплоотдачи $\alpha \approx 20,8$ ккал/ $\mathrm{m}^{2}$ час. град. Объемный коэффициент теплоотдачи $\alpha_{v \varepsilon}$ находится в пределах 1255 $1260 \mathrm{\kappa кал/} / \mathrm{M}^{3}$. час. град.

Сопоставление результатов расчета по уравнению (3) с полученными данными подтверждает принципиальную правильность последних.

Определенная таким путем величина активной поверхности теплообмена $S_{d}$ не идентична удельной поверхности $S$, теоретически вычисленной по любому из приведенных в таблице 3 методов. Она составляет примерно $0,66 S$ (таблица 3 стр. 2). Следовательнө, в шахте полукоксования из участия в процессе теплообмена выключено около $34 \%$ от интегральной говерхности кусков слоя, вследствие их контактирования между собой и, возможно, частичного слеживания $\left({ }^{20}\right)$.

Для данных условий величину активной тепловоспринимающей поверхности кускового сланца в общем виде можно выразить следующим образом:

$$
S_{\mathrm{a}}=0,66 S=\frac{4,63(1-m)}{d_{\mathrm{cp}}} .
$$

Фактически парогазовый теплоноситель весьма неравномерно распределяется по сечению шахты полукоксования. В упрощенном представлении до $30-32 \%$ от площади рабочего сечения шахты не омывается теплоносителем, так как примерно одна треть загрузки не прогревается до температуры полукоксования. Все же простые подсчеты показывают, что вследствие низкой теплопроводности сланца ( $i_{c}=0,25 \mathrm{\kappa кал/м.} \mathrm{час.}$ град.) центральная часть загрузки не может быть прогрета до температуры $210-320^{\circ}$ только за счет кондуктивной передачи тепла и лучеиспускания. Несомненно, известную роль здесь играет конвективная теплоотдача, хотя и в значительной степени ослабленная.

Поэтому при оценке интенсивности теплообмена в шахте полукоксования представляется правильным основываться на усредненных данных, хотя это и вносит некоторую погрешность в результаты расчета.

Известная погрешность в результатах расчетов обусловлена также отсутствием достаточно точных и достоверных данных о теплофизических свойствах сланца и об их изменении при различных температурах.

\section{Заключение и выводы}

В процессе теплообмена в шахте полукоксования сланцевого генератора доминирующую роль играет теплоотдача за счет вынужденной конвекции; критерием оценки интенсивности процесса является величина коэффициента конвективной теплоотдачи. 
Представляется возможным рекомендовать ряд конкретных способов определения этой величины как основы для приближенного теплового расчета при проектировании шахтных генераторов.

Сопоставление результатов решения внешней и внутренней задачи позволяет дать расчетную формулу

$$
S_{\mathrm{a}}=4,63 \frac{(1-m)}{d_{\mathrm{cp}}}=\frac{2,78}{d_{\mathrm{cp}}} \text { при } m \approx 0,4
$$

для приближенного определения величины активной тепловоспринимающей поверхности слоя технологического сланца в условиях шахты полукоксования.

Анализ основных уравнений теплообмена показывает, что дальнейшая интенсификация процесса теплоотдачи в основном может быть достигнута путем повышения степени подогрева теплоносителя, увеличения скорости и улучшения условий распределения его в слое кускового материала.

\author{
Ннститут химии \\ Академии наук Эстонской ССР
}

\author{
Поступила в редакцию \\ 9 VII 1955
}

\title{
ЛИТЕРАТУРА
}

1. И. М. А р ю хов, Пути улучшения работы сланцевого газогенератора, «За экономию топлива», № 11, стр. 1, 1951.

2. Р. С. Б е р н ш т й н, Теплоотдача в слое. Сборник «Исследование процессов горения натурального топлива», Госэнергонздат, стр. 88-97, 1948.

3. М. Я. Губергриц, О размерной характеристике кускового сланца, Известия АН ЭССР, т. ІІ, стр. 384, 1953.

4. М. Я. Г у бе рг ри ц, Л. П. Паальме. Процесс газообразования в шахтном сланцевом генераторе. Сборник Института химии АН ЭССР «Горючие сланцы», вып. 2, 1956.

5. М. Я. Гу бергриц, С. Л. Эпштейн, О. В. С амонов, А. А. Мильк, Технологический режим шахтных сланцевых генераторов. Сборник Института химии АН ЭССР «Горючие сланцы», вып. 2, 1956.

6. Д. Б. Ги н зб ург, Газификация низкосортного топлива, Промстройиздат, 1950.

7. Б. И. К и т а е в, Теплообмен в шахтных печах, Метиздат, 1945.

8. Б. И. К и т а е в, Теплообмен в доменной печи, Метиздат, 1949.

9. И. Е. К оробчан ски й, М. Д. К узнец ов, Расчеты аппаратуры для улавливания химических продуктов коксования, Метиздат, 1952.

10. М. А. Ми хе е в, Теплопередача, Госэнергоиздат, 1950.

11. К. И. С ы сков, Теория поведения кокса в доменном процессе, Изд. АН СССР, 1949.

12. В. Н. Т и м о фе ев, Теплообмен в слое, Известия ВТИ, № 2, 1949.

13. Х. К. Т р у у, О природе и свойствах слан̆ца-кукерсита, Известия АН ЭССР, т. ІІІ, cтр. $389,1954$.

14. И. М. Фед о р.ов, Теория и расчет процесса сушки, Госэнергоиздат, 1955.

15. А. Ф. Ч удн в в ки й, Теплообмен в дисперсных средах, Гостоптехиздат, 1954.

16. 3. Ф. Ч у х а нов, Е. А. Ш а п а т и н а, Дннамика процессов шевелевания топлива, Известия АН СССР, ОТН, № 4, 1946 и № 7-8, 1948.

17. J. M. C onder c, Les schistes bitumineux des liaix en Würtemberg du Sud, Oil Shale and Cannel Coal, Vol. 2, p. 38, London 1951.

18. C. C. Furn a s, Heat Transfer from a Gas Stream to a Bed of Broken Solids, Bulletin Bureau of Mines USA, No 361, 1932.

19. K. Luts, Der estländische Brennschiefer-Kukersit, seine Chemie, Technologie u. Analyse, Revaler Buchverl., 1944.

20. J. Weisman, Ch. F. B onilla, Liquid-Gas Interfacial Area in Packed Columns, Ind. Eng. Chem. Vol. 42, No 6, p. 1099, 1950. 